\title{
Metamaterial inspired THz antenna for breast cancer detection
}

\author{
G. Geetharamani ${ }^{1} \cdot$ T. Aathmanesan ${ }^{2} \mathbb{D}$ \\ (c) Springer Nature Switzerland AG 2019
}

\begin{abstract}
Metamaterial inspired Terahertz ( $\mathrm{THz}$ ) antenna for breast cancer detection proposed in this paper. The proposed antenna consists of a simple rectangular patch configuration integrated with Complementary Split Ring Resonator (CSRR). The design equations along with the equivalent circuit and permittivity calculations also presented in this paper. The experimental technique for detection of tumor in human breast model by the simulation technique also presented. The proposed metamaterial inspired antenna operates at $1 \mathrm{THz}$ frequency with $20 \mathrm{dBi}$ of gain. Its design evolution process, parametric study, and results such as farfield radiations also presented in this paper.
\end{abstract}

Keywords Metamaterials - Terahertz antenna - CSRR - Breast cancer detection.

\section{Introduction}

Researchers around the world are working to find better ways to prevent, detect, and treat breast cancer, and to improve the quality of life of patients and survivors. Therefore, it is more significant to advance the cancer diagnostic systems for better detection and treatment. Breast cancer is one among the common cancer in which the classical approach for detection includes mammography technology which must advance with the new technology for improving the diagnosis accuracy. Therefore, in this paper a new metamaterial inspired $\mathrm{THz}$ antenna for breast cancer detection presented which can more accurately detect the presence of tumor in the breast tissue. $A$ detailed study on breast cancer tumor size, overdiagnosis and mammography screening effectiveness discussed in [1]. A need for optimal breast cancer screening with personalized approach that integrates patient specific and age dependent metrices of cancer risk with selective application of specific screening technologies discussed in [2]. A combination of thermal and Electromagnetic (EM) analysis of breast cancer detection by using the surface temperature variation and perturbation concepts discussed in [3]. A study to evaluate a phyllodes tumor of the breast with magnetic resonance imaging (MRI) and magnetic resonance spectroscopy along with a detailed case study discussed in [4]. Breast cancer detection using the microwave radar technique discussed in [5-8]. A smart antenna array-based microwave imaging system for brain cancer detection focuses on tumor position discussed in [9]. A screening system for antenna array based on microwave breast cancer detection with five antennas to identify the development of malignant tissue in the women breast discussed in [10]. A flexible mild microwave hyperthermia antenna application for chemothermotheraphy of the breast discussed in [11].

A novel compact Side Slotted Vivaldi Antenna (SSVA) for microwave breast imaging discussed in [12] which includes compound radar, Integrated microwave imaging radar, Time domain multistate radar systems. The conventional medical imaging techniques uses X-rays, Magnetic resonance imaging, Ultrasound, Computer tomography and positron emission which are of low resolution and high cost for implementation and complex systems in which X-rays used in mammography for detection of detection of breast tumor where a high false negative

T. Aathmanesan, cegnesan@gmail.com | 'Department of Mathematics, Anna University, Chennai, Tamilnadu, India. ${ }^{2}$ Department of ICE, Anna University, Chennai, Tamilnadu, India.

SN Applied Sciences (2019) 1:595 | https://doi.org/10.1007/s42452-019-0601-6 
rate of (4-34\%) and high false positive rate of (70\%) have reported [13]. The potential of $(0.3-0.5 \mathrm{THz})$ frequencies in the cancer detection near field imager discussed in [14]. $\mathrm{THz}$ reflection imaging to distinguish between cancer and non-cancer breast tissue discussed in [15]. A Double Debye model to understand the dielectric response of biological tissues at $\mathrm{THz}$ frequencies especially at breast cancer discussed in [16]. An apparatus which can operate on $1.89 \mathrm{THz}$ to locate cancer in the human breast tissue discussed in [17]. A novel microstrip patch antenna on photonic crystal in THz discussed in [18]. Analysis of photonic crystal and multi-frequency terahertz microstrip patch antenna discussed in [19]. Theoretical analysis including the acquisition speed, spatial resolution, Signal to Noise Ratio (SNR), Dynamic range, Depth of field, Noise equivalent power discussed for the state-of-the-art $\mathrm{THz}$ antenna technology for imaging applications discussed in [20]. The Metamaterial concept first introduced by Veselago in the year 1968 [21]. Analytical design procedure of CSRR presented along with its equivalent circuit design discussed in [22]. An experimental demonstration of optically thin metamaterial resonating in the $\mathrm{THz}$ regime with the $70 \%$ of maximum resonance amplitude achieved by using half skin depth thick lead $(\mathrm{Pb})$ split-ring resonators (SRR) array discussed in [23]. The THz spectrum lies between Infrared (IR) and Microwave and it finds many applications in Biology, Medicine, Imaging, Security etc., and a brief review of metamaterials in $\mathrm{THz}$ region such as metamaterial absorbers, modulators, switches, lenses, and cloaking structures have discussed in [24].

Some state -of-the- art microwave breast cancer detection systems discussed in [6, 25-30]. A biomedical microwave tomography system with 3D-imaging capabilities and an accurate recovery of 3D dielectric property distributions for breast-like phantoms with tumor inclusions utilizing both the in-plane and new cross-plane data in [25]. A novel compound imaging approach presented to overcome these physical constraints and improve the imaging capabilities of a multistatic radar imaging modality for breast scanning applications discussed in [6]. A microwave imaging system developed as a clinical diagnostic tool operating in the 3- to $8-\mathrm{GHz}$ region using multistatic data collection in [26]. A second-generation monostatic radar system to measure microwave reflections from the human breast presented and analyzed in [27]. a monostatic radar-based approach to microwave imaging and utilizes ultra-wideband signals used microwave breast imaging system used to scan a small group of patients discussed in [28]. An ultrawideband (UWB) radar-based breast cancer detection system, which is composed of complementary metal-oxide-semiconductor integrated circuits, presented in [29]. The detectability of a handheld impulse-radar breast tumor detector demonstrated in [30]. From the literature review, there are techniques for detection of tumors in breast tissue using the microwave frequency were mostly available and the use of $\mathrm{THz}$ frequency is evolving gradually and the need for research in developing a $\mathrm{THz}$ based cancer detection system is increasing. Therefore, in this paper metamaterial inspired $\mathrm{THz}$ antennas for breast cancer detection presented. The organization of this paper consists of introduction with the literature review in Sect. 1 and its evolution of design along with the design methodology of the CSRR in the Sect. 2. Section 3 consists of the breast cancer detection experiment using the sample breast tissues with its results and discussion, and the Sect. 4 concludes the paper.

\section{Design of metamaterial inspired THz antenna}

\subsection{Antenna design}

The aim of this investigation was to develop a metamaterial inspired $\mathrm{THz}$ antenna for breast cancer detection. The proposed antenna shown in Fig. 1 comprise a simple rectangular patch antenna with $50 \Omega$ inset feeding in front and metamaterial inspired rectangular split ring resonator structure at the back side with width of Ws and length of $L s$ dimension and a perfect electric conductor (PEC) substrate Ts thickness Tp thickness copper over the substrate. The dimension of the patch antenna given in Table 1.

\subsection{Evolution of design}

The evolution of antenna design comprises four major steps shown in Fig. 2 in which initially, at step 1 is a simple rectangular patch with the patch length $L p$ and patch width Wp designed. The length and width of the patch taken for initial consideration since it greatly affects the resonant frequency of the antenna at first step $0.6 \mathrm{THz}$ resonant frequency with the return loss of $-11.59 \mathrm{~dB}$ along with $-10 \mathrm{~dB}$ bandwidth of $0.16 \mathrm{THz}$ obtained.

The next step 2 consists of the parametric study for modifying the ground structure for the addition of SRR structure. In step 1 the ground plane consists of gold with Ls length and Ws width and Tg thickness designed for operating in $1 \mathrm{THz}$ frequency. The parameter Ws initially modified and simulation done and there are no major changes found in the antenna. Since the parameter $\mathrm{Tg}$ is the thickness of the gold which initially fixed the next parameter is Ls is still available for parametric study. In step 2 the parametric study done with Ls and simulations done by reducing the value to $\mathrm{Ls} / 2$ and found there is improvement in return loss then $\mathrm{Gl}$ found to be the best possible length to obtain $-15.62 \mathrm{~dB}$ return loss 


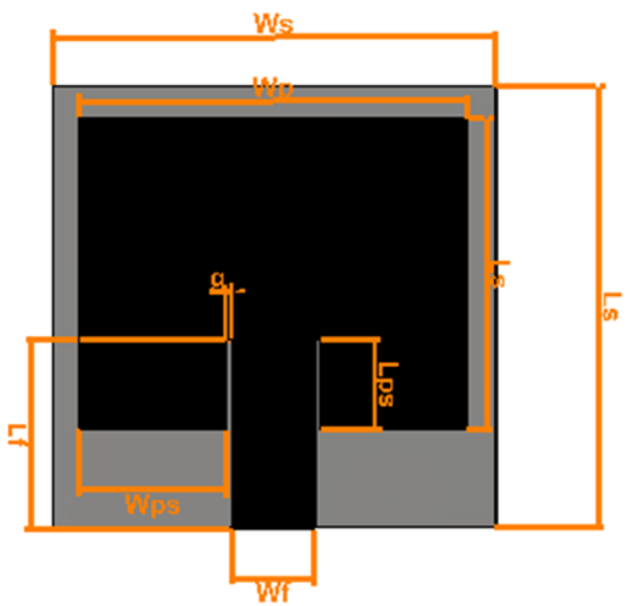

(a)

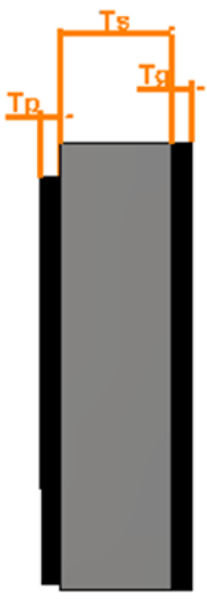

(b)

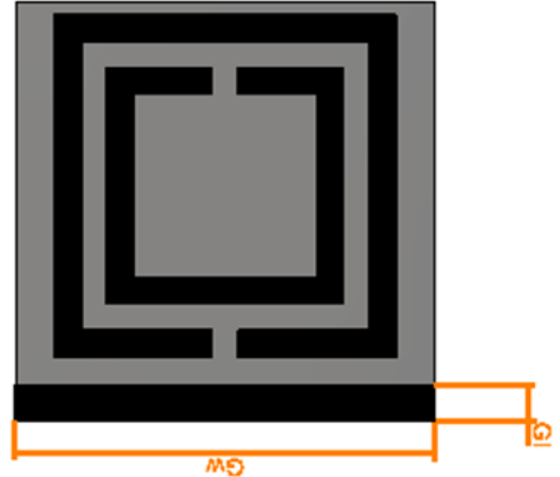

(c)

Fig. 1 Proposed antenna with its geometry

Table 1 Dimensions
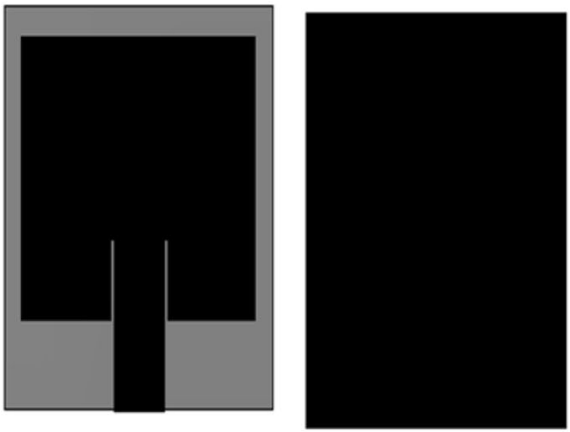

Step 1

\begin{tabular}{ll}
\hline Parameter & Dimension in $\mathrm{nm}$ \\
\hline Ws & 800 \\
Ls & 800 \\
Wp & 700 \\
Lp & 560 \\
Lf & 340 \\
Wf & 150 \\
g & 10 \\
Wps & 265 \\
Lps & 160 \\
Ts & 200 \\
Tp & 36 \\
Tg & 36 \\
Gl & 68.55 \\
Gw & 800 \\
\hline
\end{tabular}

at $0.65 \mathrm{THz}$ resonant frequency. SRR added in the step 3 which changes the current flow of a rectangular patch and changes the resonant frequencies to $0.8 \mathrm{THz}$ with the return loss of $-13 \mathrm{~dB}$ along with $-10 \mathrm{~dB}$ bandwidth of 30 $\mathrm{THz}$ respectively. Finally, at step 4 resonant frequency of 1 $\mathrm{THz}$ with return loss of $-35 \mathrm{~dB}$ along with $-10 \mathrm{~dB}$ bandwidth of $0.37 \mathrm{THz}$ the return loss plot obtained during the evolution of design and parametric study given in Fig. 3 .

The CSRR structure used here shown in Fig. 4 and its equivalent circuit with dimensions tabulated in a Table 2 .

The performance of metamaterial depends on the material properties retrieved using waveguide-based retrieval method which is effective and robust for measuring the complex permittivity and permeability of metamaterial. A waveguide port in Computer Simulation Technology (CST) is equivalent to the semi-infinite waveguide

Fig. 2 Evolution of design

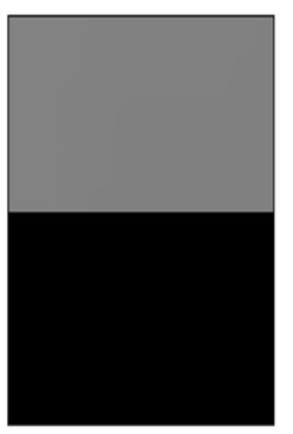

Step 2

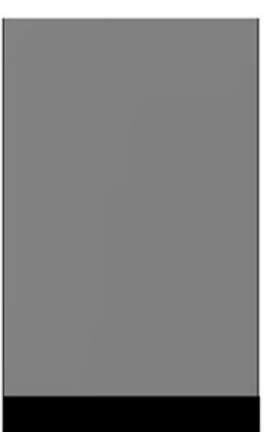

Step 3

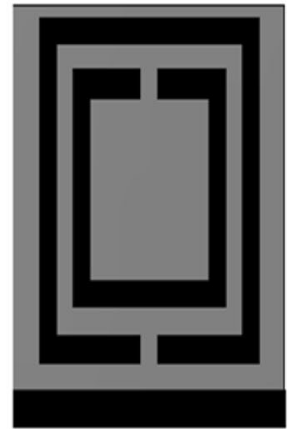

Step 4 


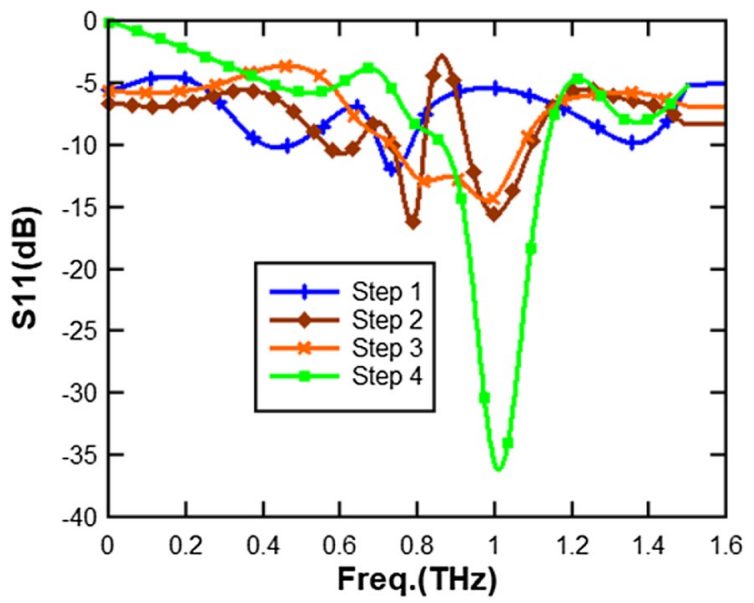

Fig. 3 Return loss observed during evolution process and parametric study

setup which can excite the structure with the incident wave perpendicular to the surface of the port. CSRR analyzed using the waveguide simulation setup shown in Fig. 5.

CSRR can act as a resonant magnetic dipole which can be excited by an axial electric field therefore, a waveguide setup with the height of air box which is 1.5 times of the substrate height for analyzing the metamaterial property of the CSRR. Perfect electric conductor (PEC) and perfect magnetic conductor (PMC) are the symmetric boundaries which used in this simulation of unit cell for creating a periodic boundary condition for analyzing the CSRR.PEC used where the magnetic field is symmetric and an electric field is asymmetric and PMC used where the electric field is symmetric and magnetic field is asymmetric. In this method CSRR kept inside the waveguide medium along $x y$ plane and the perfect electric and magnetic conductor (PEC \& PMC) fields placed on the top and bottom of the waveguide along $z$ plane and the electromagnetic field applied its input port 1 and the

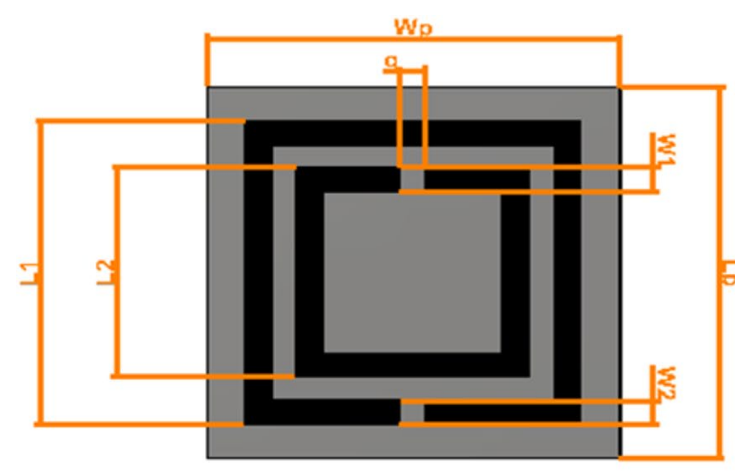

Table 2 Dimensions of the CSRR

\begin{tabular}{ll}
\hline Parameter & $\begin{array}{l}\text { Dimen- } \\
\text { sion in } \\
\text { nm }\end{array}$ \\
\hline Wp, Lp & 800 \\
L1 & 650 \\
L2 & 450 \\
W1, W2, g & 50 \\
\hline
\end{tabular}

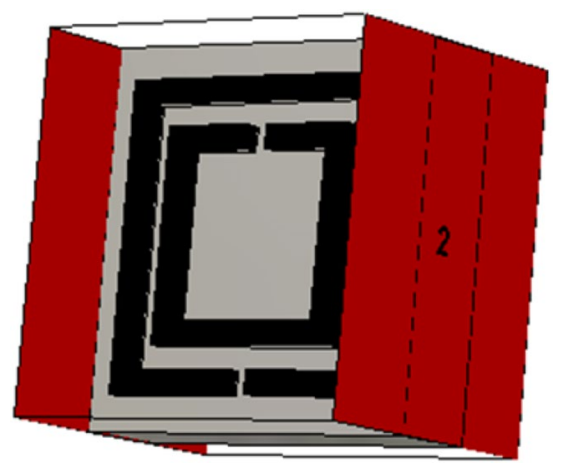

Fig. 5 Waveguide setup of CSRR simulation

corresponding S11 and S21 obtained at the output port 2 and the resonant frequency calculated from the equations given below.

$$
\begin{aligned}
& f_{C S R R}=\frac{1}{2 \pi \sqrt{L_{C S R R} C_{C S R R}}} \\
& C_{C S R R}=\frac{N-1}{2}[2 L-(2 N-1)(W+S)] C_{0} \\
& C_{0}=\varepsilon_{0} \frac{k\left(\sqrt{1-k^{2}}\right)}{k(k)}
\end{aligned}
$$

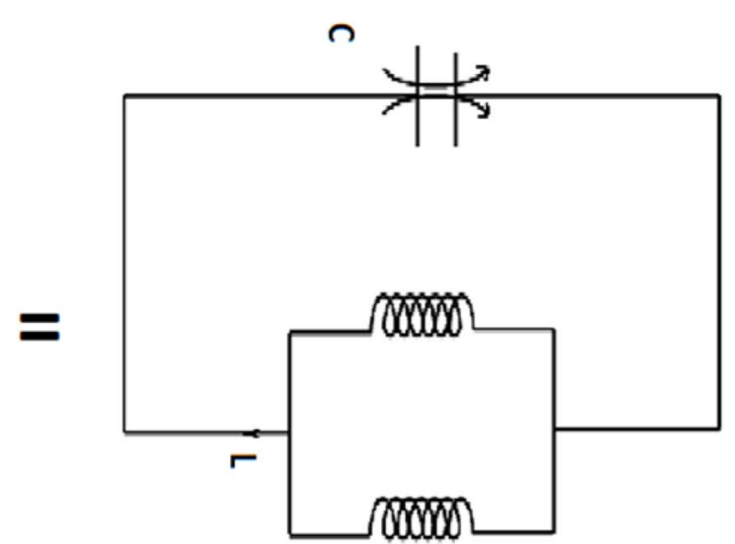

Fig. 4 CSRR along with its equivalent circuit 
$\mathrm{K}=\frac{\mathrm{s} / 2}{w+s / 2}$

$L_{C S R R}=4 \mu_{0}[L-(N-1)(S=W)]\left[\ln \left(\frac{0.98}{\rho}\right)+1.84 \rho\right]$

$\rho=\frac{(N-1)(W+S)}{1-(N-1)(W+S)}$

Here the number of CSRR is 2 and length of the rings $\mathrm{L} 1$ and $\mathrm{L} 2$ are $650 \mathrm{~nm}$ and $450 \mathrm{~nm}$, respectively. Spacing between the slots were $50 \mathrm{~mm}$ and the values of capacitance and an inductance of CSRR $L_{C S R R}$ and $C_{C S R R}$ found from the equations, For $N=2, L=650 \mathrm{~nm}$, the $f_{C S R R}$ found as $1 \mathrm{THz}$.

The negative permittivity versus frequency plot of the CSRR shown in Fig. 6 which calculated using the above equations from that the designed CSRR is resonating exactly at the resonant frequency $1 \mathrm{THz}$ since the real and imaginary part of the permittivity values was overlaps at $1 \mathrm{THz}$ it denotes the resonant frequency of the designed CSRR. The proposed antenna is excited by 50 ohms of impedance micro strip line inset feed since it provides better impedance matching and the dimensions of micro strip line feed is $340 \mathrm{~nm}$ length and $150 \mathrm{~nm}$ width. In the CST simulation environment, discrete port used for the excitation purpose.

\subsection{Performance evaluation}

Performance of the proposed metamaterial antenna analyzed in this section. Voltage standing wave ratio (VSWR) an important parameter in antenna design and VSWR for the proposed metamaterial antenna given in Fig. 7 the

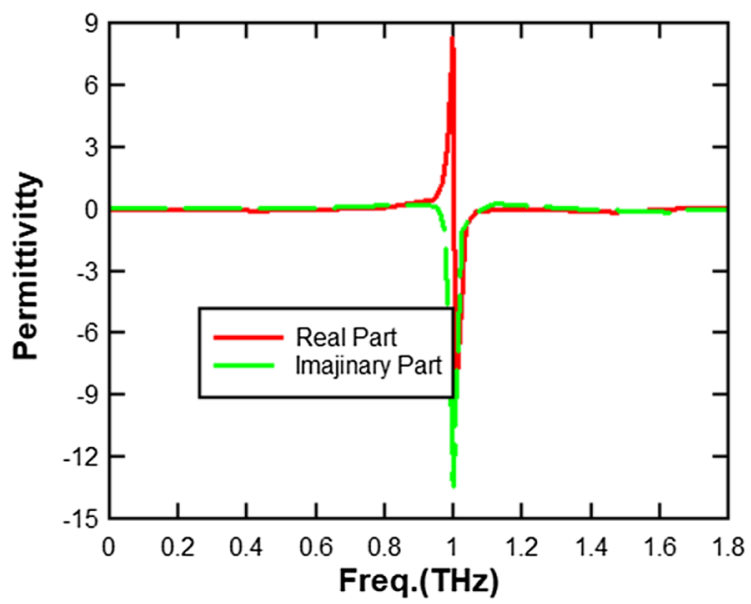

Fig. 6 permittivity versus frequency

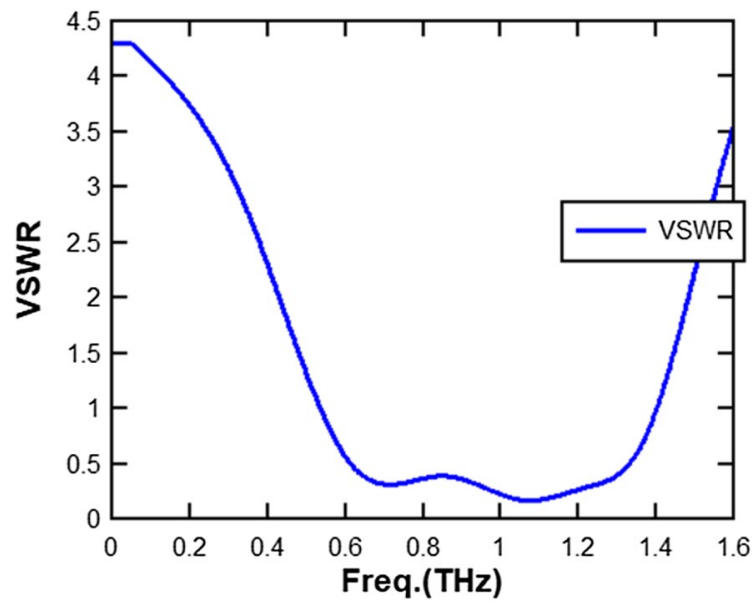

Fig. 7 VSWR of proposed metamaterial antenna

value lies below 0.5 and for the better performance VSWR expected between 0 to 1.5 and the proposed metamaterial antenna satisfies this condition (Fig. 8).

3D farfield pattern obtained from the simulation of the proposed metamaterial antenna given in Fig. 9 at $1 \mathrm{THz}$ resonant frequency shows the proposed antenna achieves $20 \mathrm{dBi}$ of gain and stable radiational characteristics over the entire operating region.

2D farfield pattern analysis plays vital role in performance analysis of any antenna. In Fig. $9 \mathrm{E}$ and $\mathrm{H}$ plane of the antenna with and without the addition of metamaterial structure at $1 \mathrm{THz}$ resonant frequency given which clearly shows that the radiational characteristics improved by the addition of the CSRR metamaterial structure. Radiations at both the $\mathrm{E}$ plane and $\mathrm{H}$ plane are highly becomes directional after the addition of metamaterial structure.
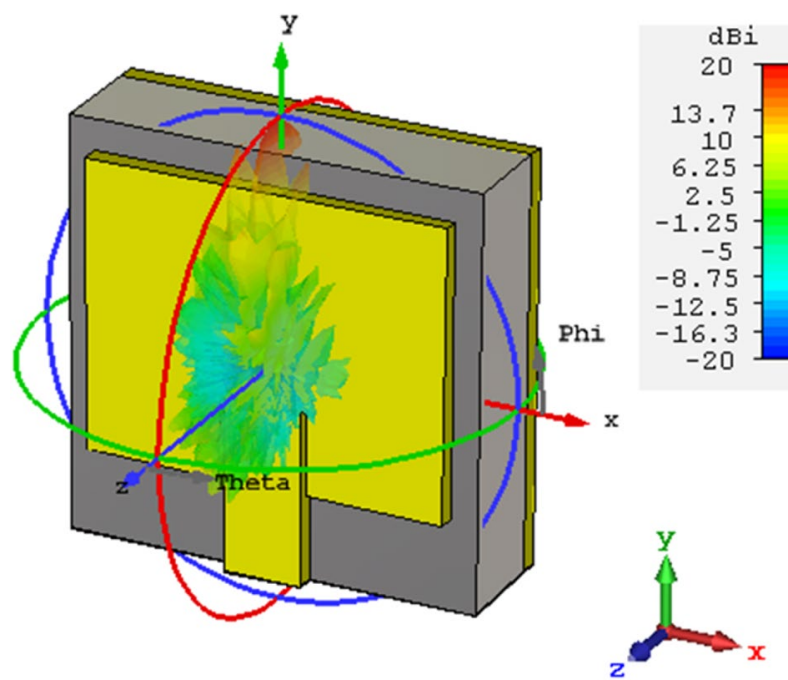

Fig. 8 3D farfield pattern of the metamaterial antenna 
Fig. 9 2D Farfield pattern of the metamaterial antenna, a antenna without metamaterial, b antenna with metamaterial

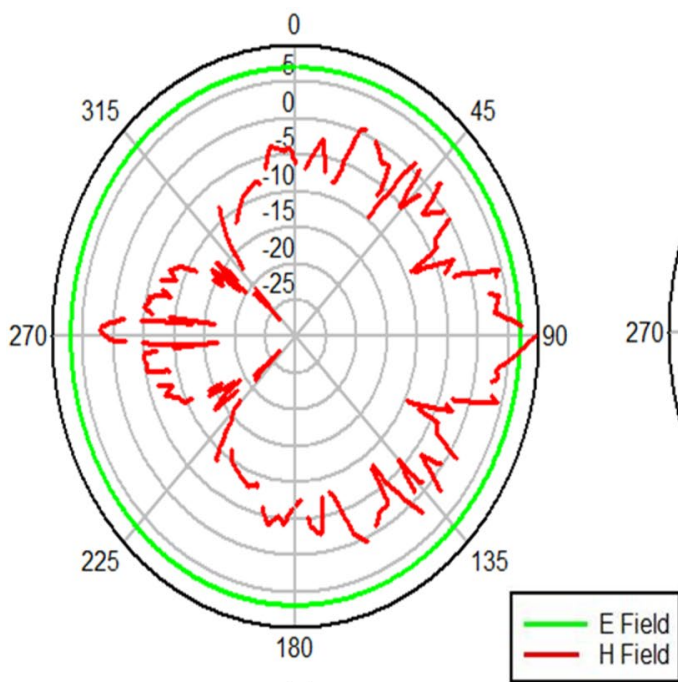

(a)

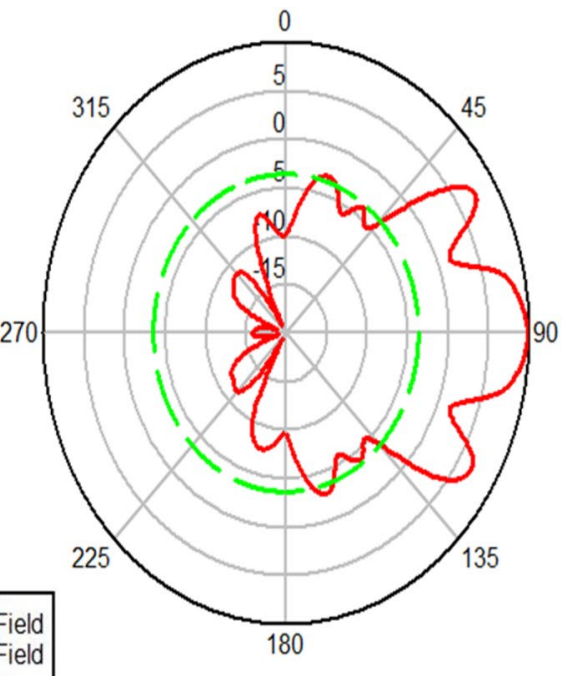

(b)

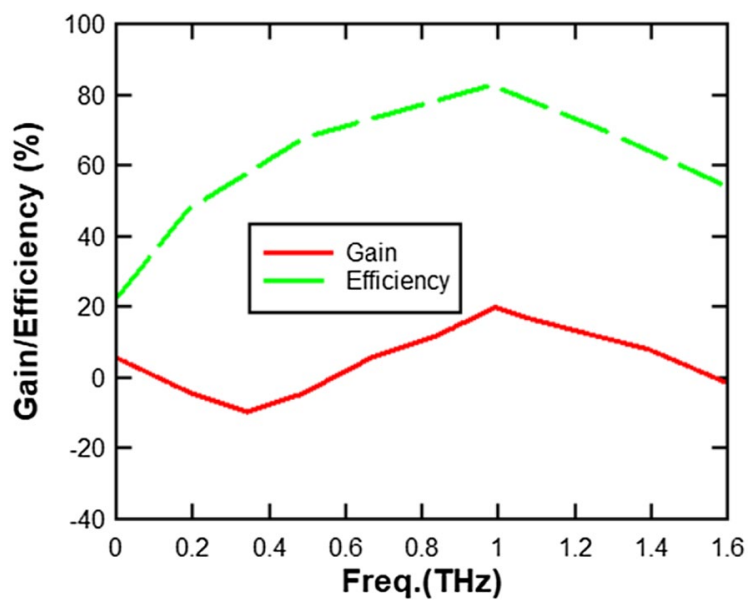

Fig. 10 Gain and efficiency versus frequency of the proposed metamaterial antenna

Gain and Efficiency versus Frequency of the proposed metamaterial antenna given in Fig. 10 shows that the antenna achieved stable gain and efficiency over the operating frequency. Gain of $20 \mathrm{dBi}$ and efficiency of $80 \%$ achieved by the proposed metamaterial antenna.

\section{Experiment}

The experimental setup for the detection of tumor in human breast model done using CST microwave studio. The physical basics of THz based breast cancer detection work as finding the difference between the normal tissue and the malignant tissue by the variation in the dielectric properties of the normal and malignant tissue. The dielectric properties of the skin can be significantly higher than

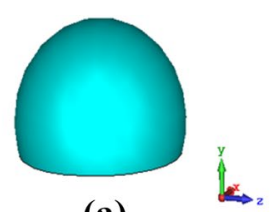

(a)
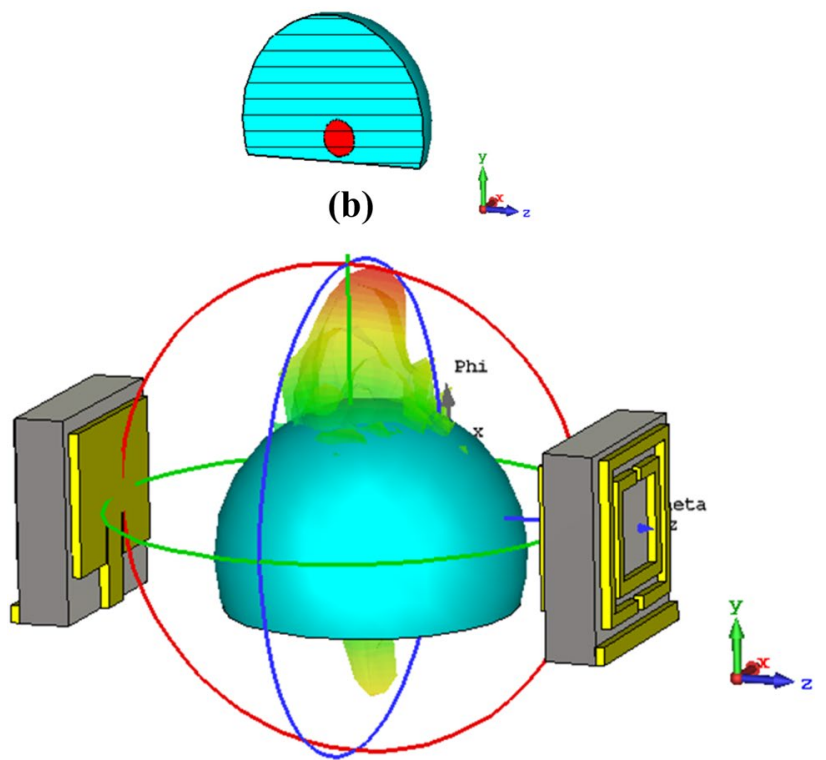

(c)

Fig. 11 a Human breast model, b human breast model along with tumor, $\mathbf{c}$ breast cancer detection experimental setup

the internal breast tissues generating unwanted reflections and multipath effects [6]. These effects analyzed and the malignant tissue identified with help of the impulse response of the antennas used in this proposed experiment. The human breast model given in Fig. 11a modelled 
using relative permittivity of 2.41 and the tumor with relative permittivity 3.18 the values of relative permittivity obtained from [31] placed inside the breast model given in Fig. 11b. The experimental setup for detecting tumor in breast tissue given in Fig. 11c. The dimension of the breast sample used in this sample is $800 * 1200 \mathrm{~nm}$ which not followed real dimension since the small sample dimension of breast tissue used here will reduce computational time and resources required for simulation and obtains faster analysis. The two metamaterial antennas act as transmitter (antenna 1) and receiver (antenna 2) antennas placed either side of the breast model along with tumor.

The experiment repeated two times for observing the signal from the breast tissue both with and without tumor for the detection of variation in the signal levels. Figure $12 \mathrm{a}$ represents the experimental setup without tumor and Fig. 12b.
In Fig. 12a power pattern without tumor given which shows the uniform distribution of transmitted power over the breast tissue and in Fig. 12b power pattern with tumor given which shows the distribution of power in not even and some power from the radiation absorbed by the tumor and leads to deflections and variation is power distribution obtained.

The input $\mathrm{THz}$ signal in time domain is given in Fig. 13a The impulse response obtained from the antenna 2 when normal tissue is given in Fig. 13b and the Fig. $13 \mathrm{c}$ shows the response when the normal tissue contains tumor in which the deflections were observed resulting in detection of variation in dielectric constant between the normal tissue and tumor.
Fig. 12 a Experimental setup without tumor, b experimental setup with tumor
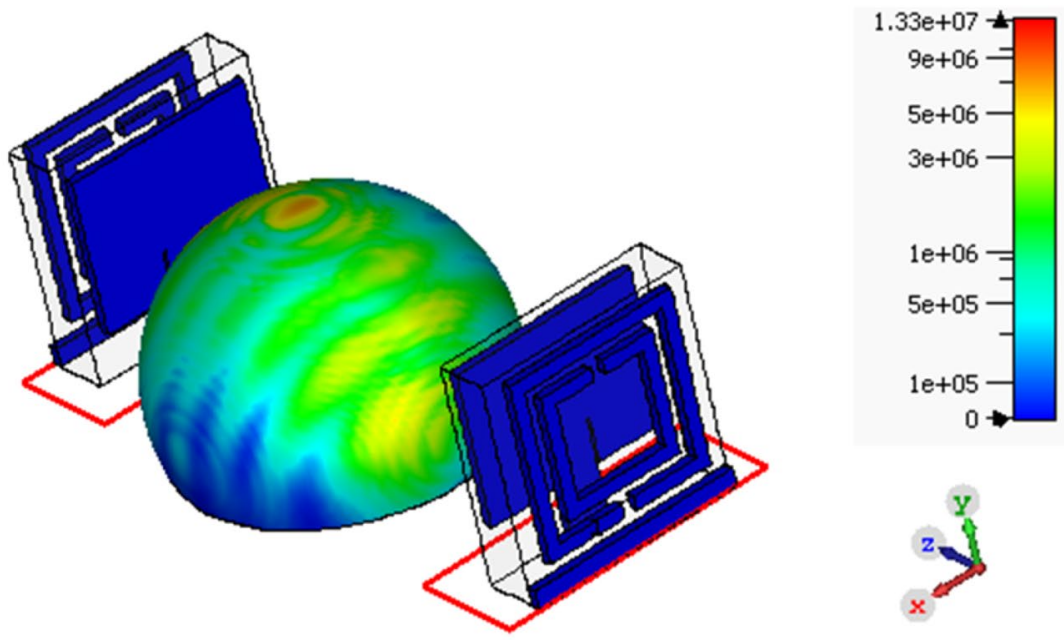

(a)
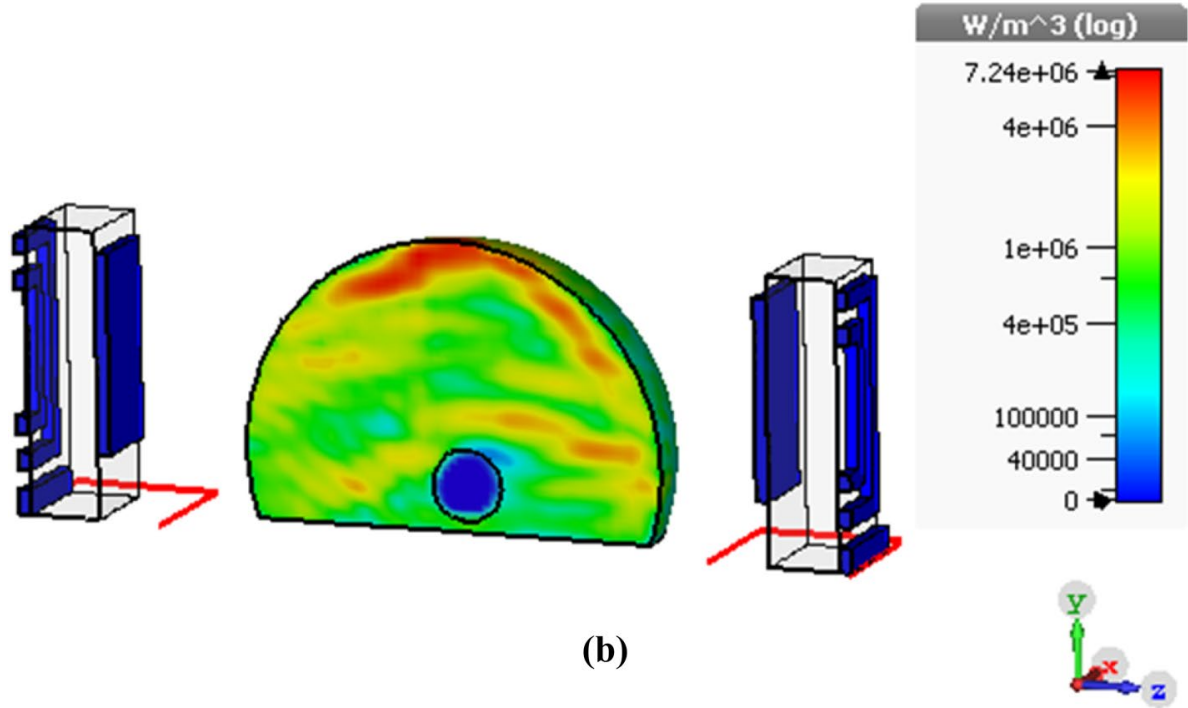

(b) 


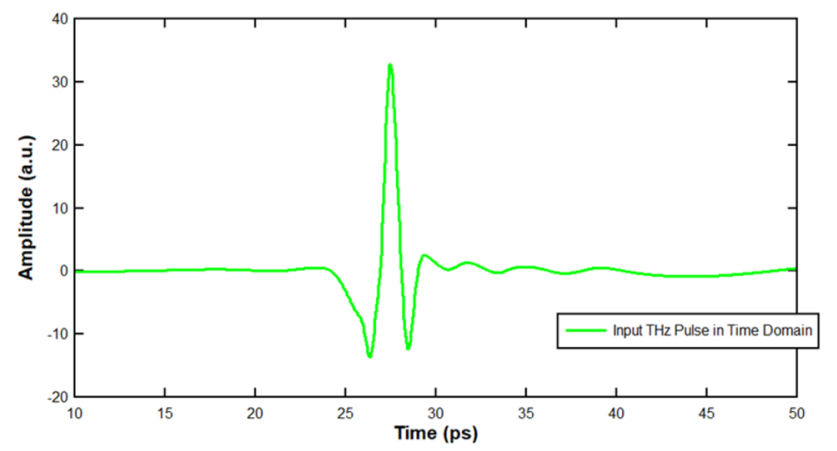

(a) Input THz Pulse

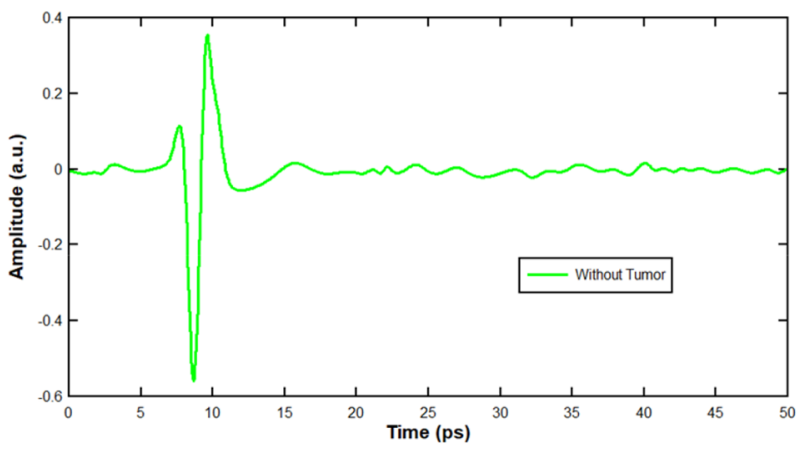

(b) Without Tumor

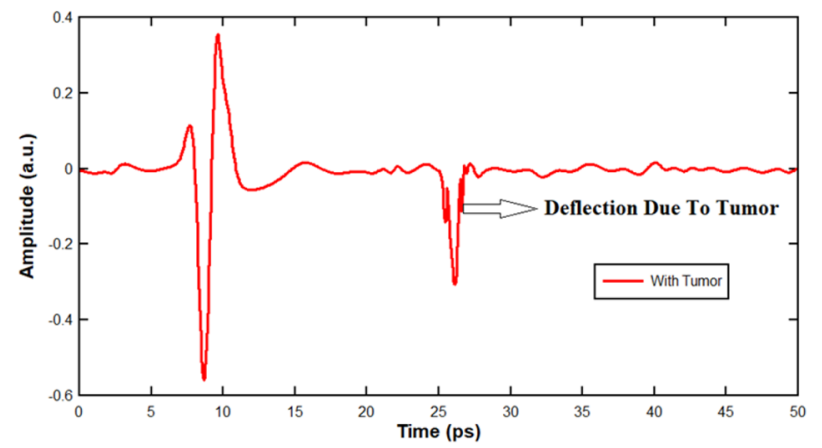

(c) With Tumor

Fig. 13 Tumor detection

\section{Conclusion}

Breast cancer is a deadly disease becoming widely common these days needs to detected in its early stages of tumor development for better treatment. This research proposes a novel method for detection of tumor in human breast tissue using metamaterial inspired antenna which operates in $\mathrm{THz}$ frequency region. The accuracy of the proposed method validated by the simulation of proposed antenna along with human breast phantom model. The experimental technique proposed in this paper clearly shows the detection of tumor in breast tissue model. The accuracy of the proposed model further improved by improving the performance characteristics of the proposed metamaterial inspired THz antenna.

\section{Compliance with ethical standards}

Conflict of interest The authors declare that they have no conflict of interest.

Human and animal rights The authors declare that there is no human participants and /or animals used in in this research

\section{References}

1. Welch HG, Prorok PC, O'Malley AJ, Kramer BS (2016) Breastcancer tumor size, overdiagnosis, and mammography screening effectiveness. N Engl J Med 375(15):1438-1447. https://doi. org/10.1056/nejmoa1600249

2. Drukteinis JS, Mooney BP, Flowers Cl, Gatenby RA (2013) Beyond mammography: new frontiers in breast cancer screening. Am J Med 126(6):472-479. https://doi.org/10.1016/j.amjme d.2012.11.025

3. Rahmatinia S, Fahimi B (2017) Magneto-thermal modeling of biological tissues: a step toward breast cancer detection. IEEE Trans Magn 53(6):1-4. https://doi.org/10.1109/tmag.2017.26717 80

4. Balaji R, Ramachandran KN (2009) Magnetic resonance imaging of a benign phyllodes tumor of the breast. Breast Care 4(3):2-2. https://doi.org/10.1159/000220604

5. Porter E, Coates M, Popovic M (2016) An early clinical study of time-domain microwave radar for breast health monitoring. IEEE Trans Biomed Eng 63(3):530-539. https://doi.org/10.1109/ tbme.2015.2465867

6. Byrne D, Sarafianou M, Craddock IJ (2017) Compound radar approach for breast imaging. IEEE Trans Biomed Eng 64(1):4051. https://doi.org/10.1109/tbme.2016.2536703

7. Bassi M, Caruso M, Khan MS, Bevilacqua A, Capobianco A-D, Neviani A (2013) An integrated microwave imaging radar with planar antennas for breast cancer detection. IEEE Trans Microw Theory Tech 61(5):2108-2118. https://doi.org/10.1109/ tmtt.2013.2247052

8. Porter E, Kirshin E, Santorelli A, Coates M, Popovic M (2013) Time-domain multistatic radar system for microwave breast screening. IEEE Antennas Wirel Propag Lett 12:229-232. https ://doi.org/10.1109/lawp.2013.2247374

9. Zhang H, El-Rayis AO, Haridas N, Noordin NH, Erdogan AT, Arslan $T$ (2011) A smart antenna array for brain cancer detection. In: 2011 Loughborough antennas and propagation conference. https://doi.org/10.1109/lapc.2011.6114045

10. Ouerghi K, Fadlallah N, Smida A, Ghayoula R, Fattahi J, Boulejfen N (2017) Circular antenna array design for breast cancer detection. In: Sensors networks smart and emerging technologies (SENSET). Beirut, pp 1-4. https://doi.org/10.1109/SENSE T.2017.8125016

11. Asili M, Chen P, Hood AZ, Purser A, Hulsey R, Johnson L, Topsakal E (2015) Flexible microwave antenna applicator for chemothermotherapy of the breast. IEEE Antennas Wirel Propag Lett 14:1778-1781. https://doi.org/10.1109/lawp.2015.2423655

12. Mahmud MZ, Islam MT, Rahman MN, Alam T, Samsuzzaman M (2017) A miniaturized directional antenna for microwave breast imaging applications. Int J Microw Wirel Technol 9(10):20132018. https://doi.org/10.1017/s1759078717000927 
13. Chandra R, Zhou H, Balasingham I, Narayanan RM (2015) On the opportunities and challenges in microwave medical sensing and imaging. IEEE Trans Biomed Eng 62(7):1667-1682. https:// doi.org/10.1109/tbme.2015.2432137

14. Al-lbadi A, et al (2017) THz spectroscopy and imaging for breast cancer detection in the $300-500 \mathrm{GHz}$ range. In: 2017 42nd International conference on infrared, millimeter, and terahertz waves (IRMMW-THz), Cancun, pp 1-1. https://doi.org/10.1109/IRMMW -THz.2017.8067037

15. Bowman TC, El-Shenawee M, Campbell LK (2015) Terahertz imaging of excised breast tumor tissue on paraffin sections. IEEE Trans Antennas Propag 63(5):2088-2097. https://doi. org/10.1109/tap.2015.2406893

16. Truong BCQ, Tuan HD, Fitzgerald AJ, Wallace VP, Nguyen HT (2015) A dielectric model of human breast tissue in terahertz regime. IEEE Trans Biomed Eng 62(2):699-707. https://doi. org/10.1109/tbme.2014.2364025

17. Peter BS, Yngvesson S, Siqueira P, Kelly P, Khan A. Glick S, Karellas A (2013) Development and testing of a single frequency terahertz imaging system for breast cancer detection. IEEE J Biomed Health Inf 17(4):785-797. https://doi.org/10.1109/ jbhi.2013.2267351

18. Kushwaha RK, Karuppanan P, Malviya LD (2018) Design and analysis of novel microstrip patch antenna on photonic crystal in THz. Phys B 545:107-112. https://doi.org/10.1016/j.physb 2018.05.045

19. Yang L, Shi X, Chen K, Fu K, Zhang B (2013) Analysis of photonic crystal and multi-frequency terahertz microstrip patch antenna. Phys B 431:11-14. https://doi.org/10.1016/j.physb.2013.08.036

20. Malhotra I, Jha KR, Singh G (2018) Terahertz antenna technology for imaging applications: a technical review. Int J Microw Wirel Technol 10(03):271-290. https://doi.org/10.1017/s175907871 $800003 x$

21. Veselago VG (1968) The electrodynamics of substances with simultaneously negative values of $s$ and $\mu$. Phys Usp 10(4):509-514

22. Baena JD, Bonache J, Martin F, Sillero RM, Falcone F, Lopetegi T, Laso MAG, Garcia- Garcia J, Gil I, Portillo MF, Sorolla M (2005) Equivalent-circuit models for split-ring resonators and complementary split-ring resonators coupled to planar transmission lines. IEEE Trans Microw Theory Tech 53(4):1451-1461. https:// doi.org/10.1109/tmtt.2005.845211

23. Singh R, Smirnova E, Taylor AJ, O'Hara JF, Zhang W (2008) Optically thin terahertz metamaterials. Opt Express 16(9):6537. https ://doi.org/10.1364/oe.16.006537

24. Choudhury M, Bisoyi S, Reddy PV, Manjula S (2014) Emerging trends in terahertz metamaterial applications. CMC Comput Mate Continua, 39(3): 179-215. https://doi.org/10.3970/ cmc.2014.039.179
25. Epstein NR, Meaney PM, Paulsen KD (2014) 3D parallel-detection microwave tomography for clinical breast imaging. Rev Sci Instrum 85(12): 124704

26. A. W. Preece, I. J. Craddock, M. Shere, L. Jones, and H. L. Winton, MARIA M4: Clinical evaluation of a prototype ultrawideband radar scanner for breast cancer detection, Journal of Medical Imaging, 3(3), 033502, 2016.29.

27. Bourqui J, Kuhlmann M, Kurrant DJ, Lavoie BR, Fear EC (2018) Adaptive monostatic system for measuring microwave reflections from the breast. Sensors 18(5):1340

28. Fear EC, Bourqui J, Curtis C, Mew D, Docktor B, Romano C (2013) Microwave breast imaging with a monostatic radar-based system: a study of application to patients. IEEE Trans Microw Theory Tech 61(5):2119-2128

29. Song $\mathrm{H}$, Kono $\mathrm{H}$, Seo $\mathrm{Y}$, Azhari A, Somei J, Suematsu E, Watarai $Y$, Ota T, Watanabe H, Hiramatsu Y, Toya A, Xiao X, Kikkawa T (2015) $A$ radar-based breast cancer detection system using CMOS integrated circuits. IEEE Access 3:2111-2121

30. Song H, Sasada S, Kadoya T, Okada M, Arihiro K, Xiao X, Kikkawa $T$ (2017) Detectability of breast tumor by a hand-held impulseradar detector: performance evaluation and pilot clinical study. Sci Rep 7(1):16353

31. Hidayat MV, Apriono C (2019) Simulation of terahertz imaging using microstrip linear array antenna for breast cancer detection. In: AIP conference proceedings, vol 2092, pp 020020

32. Yu C, Fan S, Sun Y, Pickwell-Macpherson E (2012) The potential of terahertz imaging for cancer diagnosis: a review of investigations to date. Quant Imaging Med Surg 2:33-45. https://doi. org/10.3978/j.issn.2223-4292.2012.01.04

33. Lazebnik M, Popovic D, McCartney L, Watkins CB, Lindstrom MJ, Harter J, Sewall S, Ogilvie T, Magliocco A, Breslin TM, Temple W, Mew D, Booske JH, Okoniewski M, Hagness SC (2007) A largescale study of the ultrawideband microwave dielectric properties of normal, benign and malignant breast tissues obtained from cancer surgeries. Phys Med Biol 52(20):6093-6115

34. Sugitani T, Kubota S, Kuroki S, Sogo K, Arihiro K, Okada M, Kadoya T, Hide M, Oda M, Kikkawa T (2014) Complex permittivities of breast tumor tissues obtained from cancer surgeries. Appl Phys Lett 104(25):253702

35. Martellosio A, Pasian M, Bozzi M et al (2017) Dielectric properties characterization from 0.5 to $50 \mathrm{GHz}$ of breast cancer tissues. IEEE Trans Microw Theory Tech 65(3):998-1011

Publisher's Note Springer Nature remains neutral with regard to jurisdictional claims in published maps and institutional affiliations. 